\section{How can we stimulate translational research in cancer genomics beyond bench to bedside?}

To the Editor: A huge expansion in cancer "omics" researchincluding germline and somatic mutation analysis, gene expression, epigenomics, and proteomics-is promising a new era of personalized cancer care and prevention. ${ }^{1}$ Nevertheless, to maximize the health benefits from genomic discoveries, translational research is needed to move basic science discoveriesnot only from the bench to the bedside but also to clinical and public health practice. ${ }^{2}$ Several advisory and working groups have been cautioning about the chasm between basic science discoveries and translation to population health benefits. ${ }^{3}$ Khoury et al. ${ }^{2}$ proposed four phases of translational research in genomic medicine. T1 research (bench to bedside) develops candidate health application (e.g., test or therapies). T2 research evaluates candidate applications and leads to evidence-based recommendations and guidelines. T3 research assesses how to implement and integrate an evidence-based recommendation into practice. T4 research assesses health outcomes and population impact. The authors also found that less than $2 \%$ of published genomics research between 2001 and 2006 was postbench to bedside (T2 and beyond). We recently applied this framework to a portfolio analysis of National Cancer Institute (NCI) fiscal year 2007 (FY2007) extramurally funded research in cancer genomics. ${ }^{4}$ We found that of the 1,019 funded grants, there was little funded and conducted research in the later phases of translation (T2 and beyond); only 18 grants (1.8\%) were funded in this area.

In order to evaluate the current status of translational research in cancer genomics, we updated our analysis of the NCI cancer genomics grant portfolio for FY2010. One key goal for this updated evaluation was to assess the impact of the American Recovery and Reinvestment Act of 2009 (ARRA) "stimulus" funds, which included a special request-for-application (RFA) announcement for Research and Research Infrastructure "Grand Opportunities" grants program to conduct comparative-effectiveness research in genomic and personalized medicine. ${ }^{5}$

Of the 2,137 research grants funded in FY2010 that fit our inclusion criteria, 1,989 (93.1\%) were regular non-ARRAfunded research grants, and 148 (6.9\%) were ARRA-funded. Examples of types of studies in each translation research phase are shown in Table 1. Only 36 (1.7\%) of the grants we reviewed were classified as T2 or beyond: 14 were T2, 21 were T3, and 1 grant was classified as T4. This distribution is similar to the finding from the FY2007 portfolio analysis.

However, when we stratified our analysis by type of projects, we found that among the regular non-ARRA grant pool $(n=1,989)$, only $28(1.4 \%)$ of the grants were T2 or beyond: 10 were T2, 17 were T3, and 1 was T4. On the other hand, among ARRA-funded projects $(n=148), 8(5.4 \%)$ of the grants were classified as T2 or beyond: 4 were T2 and 4 were T3. Seven of the eight ARRA grants were T2 or beyond projects that were

Table 1 The continuum of translational research in cancer genetics: types of research and examples from the portfolio analysis

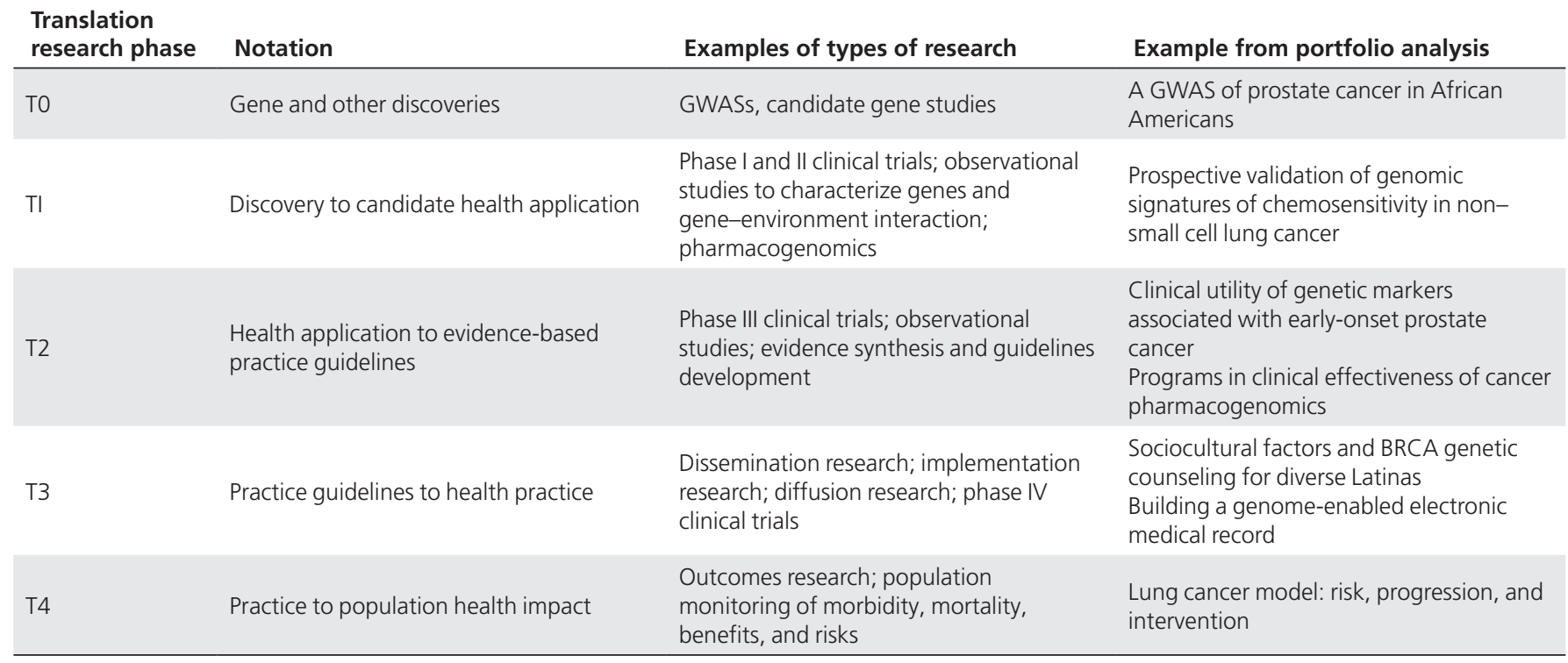

GWAS, genome-wide association study. 
funded through the Research and Research Infrastructure "Grand Opportunities" grants program.

As a result of the ARRA, in 2009, we saw stability in translational research funding beyond the bedside in our FY2010 search. However, when evaluating the NCI's general grant portfolio for FY2010, we found that only 1.4\% of cancer "omics" grants were T2 and beyond. This is a slight decrease from the $1.8 \%$ in the FY2007 grant portfolio. Because there is no new stimulus funding for this type of research on the horizon, we are concerned about the sustainability of cancer genomics translational research beyond bench to bedside.

The NCI has a strong record of funding basic science work in cancer genomics discoveries and early translation (bench to bedside) of these discoveries. This is not matched by funding to assess the value added of genomic discoveries in practice and measuring their health impact. Although our analysis highlights the continued lack of funding for translational research (beyond bench to bedside) in cancer genomics, the barriers to this type of research are unclear. Are investigators not submitting post-bedside applications to NCI? Is the National Institutes of Health review process not supportive of these types of applications? No matter what the cause, NCI will continue to find solutions to overcome the barriers to funding post-bedside research. Targeted funding announcements (such as the Clinical Sequencing Exploratory Research funding announcement released by the National Human Genome Research Institute ${ }^{6}$ ) may be one way to spark this type of research. The post-bedside translational research gap will need to be filled in order to fulfill the promise of gene discoveries for cancer care and prevention in the 21 st century.

\section{ACKNOWLEDGMENTS}

The opinions expressed in this letter are those of the authors and do not necessarily reflect the views of the Department of Health and Human Services.

\section{DISCLOSURE}

The authors declare no conflict of interest.

Sheri D. Schully, $P h D^{1}$, Camilla B. Benedicto, $M P H^{1}$ and Muin J. Khoury, MD, $P h D^{1,2}$

${ }^{1}$ Division of Cancer Control and Population Sciences, NCI, Bethesda, Maryland, USA; ${ }^{2}$ Office of Public Health Genomics, CDC, Atlanta, Georgia, USA

E-mail: schullys@mail.nih.gov

\section{REFERENCES}

1. Feero WG, Guttmacher AE, Collins FS. The genome gets personal-almost. JAMA 2008:299:1351-1352.

2. Khoury MJ, Gwinn M, Yoon PW et al. The continuum of translation research in genomic medicine: how can we accelerate the appropriate integration of human genome discoveries into health care and disease prevention? Genet Med 2007;9:665-674.

3. Institute of Medicine. Crossing the Quality Chasm: A New Health System for the 21st Century. http://www.iom.edu/Reports/2001/Crossing-the-QualityChasm-A-New-Health-System-for-the-21st-Century.aspx. Accessed 24 August 2011

4. Schully SD, Benedicto CB, Gillanders EM, Wang SS, Khoury MJ. Translational research in cancer genetics: the road less traveled. Public Health Genomics 2011;14:1-8.

5. National Cancer Institute. Comparative Effectiveness Research in Genomics and Personalized Medicine. http://cancercontrol.cancer.gov/od/phg/research. asp?type=CER. Accessed 24 August 2011

6. National Institutes of Health. Clinical Sequencing Exploratory Research (U01). http://grants.nih.gov/grants/guide/rfa-files/RFA-HG-10-017.html. Accessed 9 September 2011.

doi:10.1038/gim.2011.12 